\title{
Gonococcaemia with arthritis, dermatitis and myocarditis
}

\author{
H. S. FRASER* \\ B.SC., M.B., B.S., M.R.C.P. \\ J. P. FigueroA \\ M.B., B.S. \\ O. B. O'L. JAMES \\ M.B., B.S.
}

\author{
A. L. LIBURD \\ M.B., B.S. \\ G. A. NichOLSON \\ M.B., B.S., M.R.C.P. \\ F. WhitBOURNE \\ M.B., B.S.
}

\author{
G. A. O. Alleyne \\ M.D., F.R.C.P.
}

University Hospital of the West Indies, Mona, Kingston 7, Jamaica

\begin{abstract}
Summary
Six cases of gonococcaemia seen at the University Hospital of the West Indies are described. All presented with polyarthritis and all but one had skin lesions. They varied widely in severity and chronicity and included one case with rigors and myocarditis. Emphasis is placed on the diagnostic value of the scanty skin lesions, and the importance of repeated examination of cervical swabs.
\end{abstract}

\section{Introduction}

Gonococcaemia with arthritis and dermatitis was described in 1903 by Silvestrini. Subsequent reports, almost all from North America, distinguished two syndromes-one benign, with a mild fever, arthralgia or arthritis, and skin eruptions, and the other a serious illness with often fatal endocarditis (Levin and Silvers, 1937; Reitzel and Kohl, 1938; Williams, 1938). After the introduction of penicillin both syndromes were regarded as rarities until recent years (British Medical Journal, Editorial 1970). Abu-Nassar et al. (1963) drew attention to the specificity of the cutaneous lesion, and the arthritisdermatitis syndrome(Holmes, Wiesner and Pederson, 1971), or benign gonococcaemia (Wolff, Goodman and Vahrman, 1970), has been increasingly recognized. It is described as a uniformly mild condition responding rapidly to penicillin (Abu-Nassar et al., 1963; Barr and Danielson, 1971).

This report describes six patients in Jamaica with gonococcaemia of varying severity-two were seriously ill, including one with myocarditis. Emphasis is placed on early diagnosis.

\footnotetext{
*Present address: Department of Clinical Pharmacology, Royal Postgraduate Medical School, Hammersmith Hospital, London W12 0HS.
}

Patients and methods

Six female patients with gonococcal arthritis were admitted by a single medical firm to the University Hospital of the West Indies (UHWI) between July and December, 1972. A clinical diagnosis of gonococcaemia was made on admission in all but one patient. Attempts were made to confirm it by examination of cervical smears and joint aspirates for Neisseria gonorrhoea by Gram stain; culture of cervical swabs, joint aspirates and blood; and the Gonococcal Complement Fixation Test (GCFT) on blood and joint aspirates. Examination of skin lesions was not attempted because of the uniformly poor results reported in other series using routine methods (Abu-Nassar et al., 1963; Barr and Danielsson, 1971) and the unavailability of the immunofluorescent technique (Barr and Danielsson, 1971; Danielsson, 1965). Latex fixation, anti-nuclear factor and LE cell preparations were done on all patients and were negative except for the anti-nuclear factor in case 3. All patients gave a history of sexual intercourse within the previous 3 weeks.

Treatment was started without delay if a Gram stain revealed Gram-negative intracellular diplococci and the clinical picture was typical. The diagnosis was considered confirmed if $N$. gonorrhoea was cultured from the cervical swab, joint aspirate or blood, or if the joint aspirate GCFT was positive.

\section{Case 1}

A 25-year-old woman, 24 weeks pregnant, was admitted on $10 \mathrm{July,}$ 1972. She complained of pains in several joints over a 2-week period. The first pain was of sudden onset in the left sole and ankle and lasted 2 days, followed 4 days later by pain in the right sole and pain and swelling of the right wrist. She then noticed skin lesions on both feet. In the 
second week the swelling of the right wrist extended to the dorsum of the hand and thumb and she could not use the hand. She admitted to an abnormal yellow vaginal discharge for 3 weeks.

On admission she was ill-looking with a temperature of $101^{\circ} \mathrm{F}$ and in great pain from tenosynovitis of the right hand. There was a pustule on the left hand, three haemorrhagic pustules on the soles, and examination revealed a copious yellow vaginal discharge.

The white cell count was $9000 / \mathrm{mm}^{3}$, and the ESR $114 \mathrm{~mm} / \mathrm{hr} . N$. gonorrhoea was demonstrated in the cervical smear, and isolated on culture. The serum GCFT was positive. She was treated with analgesics, 1 million iu of crystalline penicillin 6-hourly i.m., initial splinting of the hand, and early physiotherapy. Her temperature settled rapidly, the tenosynovitis began to respond within $24 \mathrm{hr}$, and had resolved completely by the ninth day without impairment of function.

\section{Case 2}

A 22-year-old woman was admitted on 31 August, 1972 , complaining of pain and swelling of the right knee, right shoulder and the left shoulder, elbow, wrist and fingers for 2 weeks. She had felt unwell, with vomiting, and had seen two vesicles appear on the right hand at the onset of her pains; there had been an offensive yellow vaginal discharge over the previous 3 days.

She did not appear very ill on admission. She had a temperature of $100^{\circ} \mathrm{F}$, a severely tender right knee with an effusion which yielded a yellow aspirate, two resolving haemorrhagic vesicles on the right hand and minimal cervical discharge. Pain and swelling developed in the left knee on the day after admission.

The white cell count was $5600 / \mathrm{mm}^{3}$, and the ESR $127 \mathrm{~mm} / \mathrm{hr}$. The Gram stain of the cervical smear was reported as negative, and was repeated twice, showing only extracellular diplococci on each occasion. This led to a delay in treatment of 2 days, during which joint swelling increased, and $70 \mathrm{ml}$ of fluid were aspirated. $N$. gonorrhoea was grown from the aspirate and from the cervical swab. The serum GCFT was negative on admission and in the second week but the aspirate GCFT was positive.

Treatment was begun with 1 million iu of crystalline penicillin i.m. 6 hourly and her temperature settled promptly. Resolution of the swelling of the right knee was initially very slow but she was asymptomatic by the twelfth day.

\section{Case 3}

An 18-year-old schoolgirl was admitted on 9 September, 1972, with a 1-week history of a widespread rash and a painful left thumb, and sudden onset of severe pain and swelling in the left knee the day before admission. She admitted to an abnormally heavy vaginal discharge of uncertain duration.

She had been admitted 4 years previously with $\overrightarrow{\bar{F}}$ arthritis, fever and a rash, and a diagnosis of $\overrightarrow{0}$ systemic lupus erythematosus had been made on the basis of the clinical picture, a raised ESR and a positive anti-nuclear factor. Prednisone was needed to control her symptoms but she had been well and defaulted during the past year.

On examination she had a temperature of $101^{\circ} \mathrm{F}$, a pulse of $100 / \mathrm{min}$, a painful, swollen left knee held in flexion, and mild tenderness and swelling of the left ankle. There was a fine maculo-papular rash, with some larger haemorrhagic vesiculo-pustular lesions, on the hands, forearms, upper arms, thighs and chest. One of these lesions on the left thumb had a wide necrotic base. There was a copious yellowgreen vaginal discharge.

Over the first 3 days in hospital new skin lesions appeared and the left knee became grossly swollen and painful, requiring large doses of pethidine. Three attempts at aspiration were unsuccessful. Her temperature remained between $100-103^{\circ} \mathrm{F}$, her pulse between 120 and 140/min and she was ill and withdrawn. The white cell count was $11,400 / \mathrm{mm}^{3}$, the ESR $124 \mathrm{~mm} / \mathrm{hr}$, and the anti-nuclear factog strongly positive. Cervical swabs were reported as negative initially, but Gram-negative intracellulap diplococci were seen in swabs taken on the thirđ day and $N$. gonorrhoea was isolated from the first swab. She was treated with prednisone at the same time as tetracycline, $500 \mathrm{mg}$ 6-hourly. Her temperature and pulse settled in $48 \mathrm{hr}$, while all symptoms and signs in the knee took 10 days to resolve completely.

\section{Case 4}

A 40-year-old woman was admitted on 16 September with a history of pain and swelling of both knees, ankles and elbows for about 1 month. She had noticed an excessive vaginal discharge for several weeks but this had stopped a week before admission.

She was afebrile with mild swelling and tenderness of knee and ankle joints. There were no skin lesions and no significant vaginal discharge. The white cell count was $9700 / \mathrm{mm}^{3}$ and the ESR $38 \mathrm{~mm} / \mathrm{hr}$. The cervical smear showed no $N$. gonorrhoea, and was negative on culture, but blood and joint aspirate both yielded a positive GCFT.

She responded slowly to therapy with intramuscular crystalline penicillin and was discharged on oral penicillin after 14 days with no swelling, but with pain in both knees. This was still present 2 weeks later. X-rays of the knee joints showed no abnormality. 


\section{Case 5}

An obese, 35-year-old woman was admitted on 1 October, 1972, with a 12-day history of pain and swelling in her left knee and pain for 1 day in the left ankle. She had been treated by a general practitioner 3 weeks previously for a vaginitis concurrent with pain in the left hip and shoulder.

She had a temperature of $101^{\circ} \mathrm{F}$, and the left knee was tender, with an effusion which yielded a yellow aspirate. There was a maculo-papular rash over the back of her wrists, hands and arms. There were signs of pelvic inflammatory disease but no vaginal discharge.

The white cell count was 7600 and the ESR $60 \mathrm{~mm} /$ $\mathrm{hr}$. Gram stain and culture of the cervical swab were negative for $N$. gonorrhoea and culture of the joint aspirate was negative, but blood and aspirate GCFT were both positive.

She was treated with 2 million iu of crystalline penicillin intramuscularly 6 hourly for 14 days. Initially, improvement was rapid, but pain and swelling only disappeared completely when she was given a further 2-week course of oral penicillin.

\section{Case 6}

A 22-year-old woman was admitted to UHWI for the second time on 5 December, 1972, with a referral diagnosis of rheumatic fever. Her illness began 5 days previously with fever, headache and malaise. On the third day she developed pain over the dorsum of her right foot and noticed three small papules on her hands. On the day of admission she developed pain in the right elbow and left wrist, dyspnoea, palpitations, rigors and vomiting, and visited the general practitioner.

This patient had myasthenia gravis and had presented 4 months earlier with severe ptosis, diplopia and generalized weakness. She had responded poorly to anticholinesterases, but made a full recovery on prednisone. Her current therapy was prednisone $20 \mathrm{mg}$ plus $720 \mathrm{mg}$ of pyridostigmine daily.

On admission she was very ill, with a temperature of $102 \cdot 6^{\circ} \mathrm{F}$, rigors, a pulse of $140 / \mathrm{min}$ and $4-6$ extrasystoles/min. There was no evidence of endocarditis or cardiac failure. There was a pustule of $3 \mathrm{~mm}$ diameter, surrounded by a violaceous halo $1 \mathrm{~cm}$ in diameter on her left palm, one haemorrhagic papule on each hand, and two erythematous vesicles on the left arm and right thigh. The right foot was tender, warm, red and swollen over the dorso-lateral aspects of the ankle; the right elbow and wrist were painful but not swollen. There was a moderate amount of yellow vaginal discharge.

The white cell count was 30,700 , the ESR $62 / \mathrm{hr}$, and the ECG showed inverted and flattened T-waves on the chest leads with multiple ventricular extrasystoles (see Fig. 1). Blood cultures taken during rigors yielded $N$. gonorrhoea and the organisms were identified in cervical and high vaginal swabs by Gram stain. Blood GCFT was positive, but no joint aspirate was obtained.

Treatment was begun with 1 million iu crystalline penicillin i.m. 6 hourly. By the following day her temperature had fallen dramatically and the ankle tenosynovitis improved, but tachycardia and extrasystoles were still marked. In view of her prednisone medication and the evidence of myocarditis, therapy was changed to 20 million iu of crystalline penicillin daily i.v. for 14 days. The pustule on the left hand enlarged to $1 \mathrm{~cm}$ diameter and progressed to a dirty grey necrotic lesion, but finally healed leaving an area of hyperpigmentation. Other symptoms and signs disappeared completely within 5 days of treatment. She had no symptoms of myasthenia throughout this illness.

Clinical and laboratory features are summarized in the table. These six patients presented a spectrum both in severity and duration of illness, and it can be seen that the patients presenting with the shortest history were most severely ill, had highest temperatures and highest WBC and ESR, classical skin lesions, and a vaginal discharge on admission. Positive cervical smears and cultures were obtained in three out of four, effusions were too small for aspiration in three out of four, and rapid response to penicillin occurred in all. Conversely, the patients with the longest history had negative cervical swabs but sizeable effusions with a positive aspirate GCFT and they responded much more slowly to penicillin.

Skin lesions. Classical embolic lesions, ranging from haemorrhagic maculo papules to vesiculo pustules, occurred in four patients. In three they were typically sparse and situated peripherally, and in two progression to a large necrotic lesion was seen. In one of the four, lesions were generalized and occurred in three separate crops. In a fifth patient a sparse maculo-papular rash occurred on the hands and wrists which could conceivably have been a reaction to an unidentified drug. The absolute specificity of the lesions in these patients bears reemphasizing, as their significance was missed in all cases by the first doctor the patient saw.

Arthritis. The distribution of arthritis conforms to the accepted pattern of a polyarthritis and/or polyarthralgia, usually with one joint (knee, ankle or wrist) most severely involved. Tenosynovitis occurred in two patients.

Vaginal discharge. In all patients a history was obtained of recent sexual intercourse and abnormal or excessive vaginal discharge. Unlike many series where no features of venereal disease were present, four of our six patients presented with a discharge from which $N$. gonorrhoea was identified or isolated. In two of three smears yielding Gram-negative 


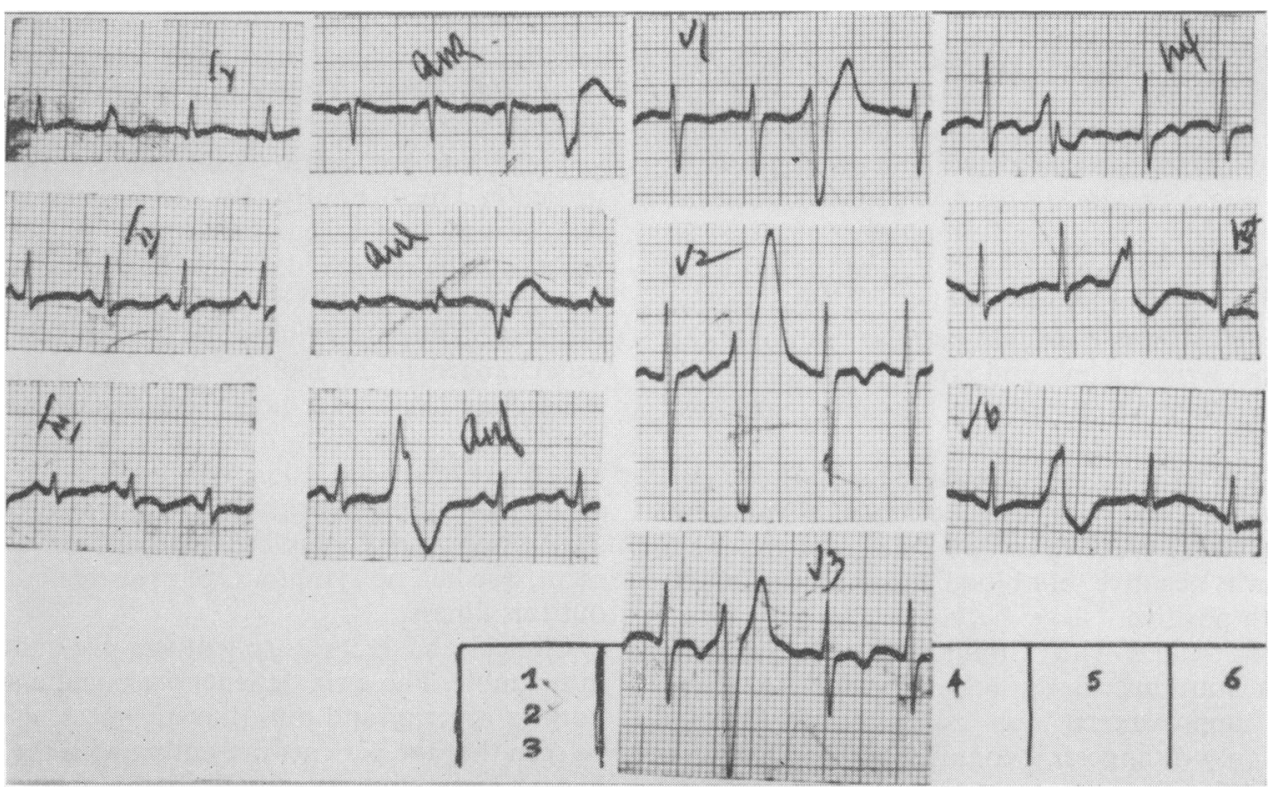

FIG. 1. ECG of Case 6 on day of admission.

TABLE 1. Relation between the duration of symptoms and the severity of clinical parameters and laboratory indices

\begin{tabular}{|c|c|c|c|c|c|c|}
\hline Case no: & 6 & 3 & 1 & 2 & 5 & 4 \\
\hline Duration of symptoms (days) & 5 & 7 & 10 & 14 & 21 & 30 \\
\hline Admission temp in ${ }^{\circ} \mathbf{F}$ & $102 \cdot 6$ & 102 & 101 & 100 & 101 & 99 \\
\hline No. of joints involved & 3 & 3 & 3 & 2 & 4 & 6 \\
\hline Vaginal discharge & + & + & + & + & - & - \\
\hline White cell count $/ \mathrm{mm}^{3}$ & 30,700 & 11,400 & 9500 & 5600 & 7600 & 9700 \\
\hline $\mathrm{ESR} \mathrm{mm} / \mathrm{hr}$ & 62 & 124 & 114 & 127 & 60 & 38 \\
\hline $\begin{array}{l}\text { Cervix smear (Gram stain) } \\
\text { Culture }\end{array}$ & \pm & $\stackrel{+}{+}$ & $\begin{array}{l}+ \\
+\end{array}$ & $\bar{t}$ & $\overline{-}$ & - \\
\hline $\begin{array}{l}\text { Joint aspirate } \\
\text { GCFT } \\
\text { Culture }\end{array}$ & $\begin{array}{l}\text { n.d. } \\
\text { n.d. }\end{array}$ & $\begin{array}{l}\mathbf{U} \\
\mathbf{U}\end{array}$ & $\begin{array}{l}\text { n.d. } \\
\text { n.d. }\end{array}$ & + & \pm & \pm \\
\hline $\begin{array}{l}\text { Blood } \\
\text { GCFT } \\
\text { Culture }\end{array}$ & $\stackrel{+}{+}$ & \pm & $\underset{\text { n.d. }}{+}$ & $\overline{\text { n.d. }}$ & $\stackrel{+}{+}$ & $\stackrel{+}{+}$ \\
\hline No. of positive specimens & $3 / 4$ & $3 / 4$ & $3 / 3$ & $4 / 5$ & $2 / 5$ & $2 / 5$ \\
\hline $\begin{array}{l}\text { Disappearance of symptoms } \\
\text { (days) }\end{array}$ & 5 & 10 & 9 & 12 & 28 & $>28$ \\
\hline
\end{tabular}


intracellular diplococci, specimens were initially interpreted as negative or extracellular, and reexamination or repeated sampling produced a positive result.

\section{Discussion}

Arthritis was a common complication of gonorrhoea in the pre-antibiotic era, occurring chiefly in males. It is once again recognized as a common disorder, perhaps in association with the rising incidence of gonorrhoea throughout the world in the last decade (Guthe, 1972). It now occurs predominantly in females (Abu-Nassar et al., 1963; Keiser, Ruben and Wolinsky, 1968) and frequently in association with skin lesions as part of a gonococcaemia (Keil, 1938; Abu-Nassar et al., 1963; Barr and Danielsson, 1971). Distinction has been made between benign gonococcaemia and a serious illness with endocarditis (Abu-Nassar et al., 1963) while Keiser et al. (1968) have divided patients into those with septic forms and those without.

However, all gradations can occur, and the severe illness with cardiac involvement should not be regarded as an illness of the pre-antibiotic area alone. One of our most ill patients had SLE, in which an increased incidence of infection is recognized (Dubois and Tuffanelli, 1964; Edelen et al., 1971) while another was being treated with prednisone. Cooke et al. (1971) describes two patients with gonorrhoea who were severely toxic after receiving steroids before hospital referral.

A rapid means of confirming the diagnosis is essential. IFA techniques give the most rapid and reliable confirmation but are not universally available. We have found cervical smears the most useful if examined promptly by experienced personnel; the need to repeat the swab if a negative result is first obtained is supported by the finding of Schmale, Martin and Domescik (1969) that $8.6 \%$ of females with gonorrhoea were negative on first sampling and positive on the second. Furthermore, in chronic cases organisms may only be seen extracellularly (Wilcox, 1964).

The GCFT on synovial fluid was consistently positive in our patients but the investigation is timeconsuming and joint fluid is not always obtainable.

Before penicillin was available, fulminating gonococcal endocarditis was not uncommon (Williams, 1938; Davis and Romansky, 1956). Gonococcal myocarditis is less well recognized. Master and Jaffe (1934) listed the ECG abnormalities of myocarditis in a wide variety of patients with acute infections, including sixteen patients with gonococcal arthritis. Five of these had a tachycardia of more than $120 / \mathrm{min}$, three had PR interval of more than $0 \cdot 2 \mathrm{sec}$, three had T-wave inversion and two had RST abnormalities. Other cases were described by Bang in 1940, and Shapiro et al. (1949) reported four cases in order to demonstrate that polyarthritis and myocarditis are not pathognomonic of acute rheumatism. In developing countries such as Jamaica, where acute rheumatism is still a common cause of morbidity and mortality, the distinction is important for early specific treatment. Furthermore, the increasing use of steroids and immunosuppressive agents in societies without comprehensive health services will inevitably produce severe complicating infections as described here.

\section{Acknowledgment}

We wish to thank Miss Elizabeth Turner and Miss Bernadette Edinburgh for their secretarial help.

\section{References}

Abu-Nassar, H., Hill, N., Fred, H.L. \& Yow, E.M. (1963) Cutaneous manifestations of Gonococcemia. Archives of Internal Medicine, 112, 731.

BANG, O. (1940) Gonorrhoeal myocarditis. British Medical Journal, 1, 117.

BARR, J. \& DANielsson, D. (1971) Septic gonococcal dermatitis. British Medical Journal, 1, 482.

British Medical Journal (1970) Complications of gonorrhoea (Editorial), 3, 420.

CoOKe, C.L., OWen, D.S., Irby, R. \& Toone, E. (1971) Gonococcal arthritis. Journal of the American Medical Association, 217, 204.

Danielsson, D. (1965) The demonstration of $N$. gonorrhoeae with the aid of fluorescent antibodies. Acta DermatoVenereologica, 45, 61 .

Davis, D.S. \& Romansky, M.J. (1956) Gonococcic endocarditis: review of literature. American Journal of Medicine, 21, 473.

Dubois, E.L. \& Tuffanelli, D.L. (1964) Clinical manifestations of systemic lupus erythematosus: computer analysis of 520 cases. Journal of the American Medical Association, $190,104$.

Edelen, J.S., Lockshin, M.D. \& Le Roy, E.C. (1971) Gonococcal arthritis in two patients with active lupus erythematosus. Arthritis and Rheumatism, 14, 557.

Guthe, T. (1972) Present status of gonorrhea control. Postgraduate Medical Journal, January Suppl., 7.

Holmes, K., Wiesner, P.J. \& Pedersen, A. (1971) The gonococcal arthritis-dermatitis syndrome. Annals of Internal Medicine, 75, 3.

KeIL, H. (1938) A type of gonococcal bacteraemia with characteristic haemorrhagic vesiculo-pustular and bullous skin lesions. Quarterly Journal of Medicine, 7, 1.

Keiser, H., Ruben, F.L. \& Wolinsky, E. (1968) Clinical forms of gonococcal arthritis. New England Journal of Medicine, 279, 234.

LeVIN, O.L. \& Silvers, S.H. (1937) Cutaneous eruptions in gonorrhea. New York Journal of Medicine, 37, 1712.

MASTER, A.M. \& JAFFE, H. (1934) Electrocardiographic evidence of cardiac involvement in acute disease. Proceedings of the Society for Experimental Biology and Medicine, 31, 931.

Reitzel, R.J. \& KoHL, C. (1938) The identification of gonococci in complications of gonorrhea. Journal of the American Medical Association, 110, 1095. 
Schmale, J.D., Martin, J.E. \& DomesciK, G. (1969) Asymptomatic gonorrhea. Journal of the American Medical Association, 210, 312.

Shapiro, E., Lipkis, M.L., KaHN, J. \& Heid, J.B. (1949) Electrocardiographic changes in acute gonococcal arthritis and myocarditis simulating acute rheumatic polyarthritis. American Journal of Medical Science, 217, 300.

Silvestrini, R. (1903) Rivista critica di clinica medica, 4, 385.
Wilcox, R.R. (1964) Textbook of Venereal Diseases and Treponematoses, p. 31. William Heinemann: London.

Williams, R.H. (1938) Gonococcic Endocarditis: A study of 12 cases, with 10 post-mortem examinations. Archives of Internal Medicine, 61, 26.

WolfF, C.B., Goodman, H.V. \& Vahrman, J. (1970) Gonorrhoea with skin and joint manifestations. British Medical Journal, 2, 271. 